\title{
Molecular mechanism of ATP versus GTP selectivity of adenylate kinase
}

\author{
Per Rogne $^{a}$, Marie Rosselin ${ }^{a, 1}$, Christin Grundström ${ }^{a}$, Christian Hedberg ${ }^{a}$, Uwe H. Sauer ${ }^{a, 2}$, and Magnus Wolf-Watz ${ }^{a, 2}$ \\ aDepartment of Chemistry, Umeå University, Umeå SE-901 87, Sweden
}

Edited by Michael F. Summers, Howard Hughes Medical Institute and University of Maryland, Baltimore, MD, and approved February 8, 2018 (received for review December 12, 2017)

\begin{abstract}
Enzymatic substrate selectivity is critical for the precise control of metabolic pathways. In cases where chemically related substrates are present inside cells, robust mechanisms of substrate selectivity are required. Here, we report the mechanism utilized for catalytic ATP versus GTP selectivity during adenylate kinase (Adk) -mediated phosphorylation of AMP. Using NMR spectroscopy we found that while Adk adopts a catalytically competent and closed structural state in complex with ATP, the enzyme is arrested in a catalytically inhibited and open state in complex with GTP. X-ray crystallography experiments revealed that the interaction interfaces supporting ATP and GTP recognition, in part, are mediated by coinciding residues. The mechanism provides an atomic view on how the cellular GTP pool is protected from Adk turnover, which is important because GTP has many specialized cellular functions. In further support of this mechanism, a structure-function analysis enabled by synthesis of ATP analogs suggests that a hydrogen bond between the adenine moiety and the backbone of the enzyme is vital for ATP selectivity. The importance of the hydrogen bond for substrate selectivity is likely general given the conservation of its location and orientation across the family of eukaryotic protein kinases.
\end{abstract}

adenylate kinase | selectivity | ATP | GTP | mechanism

$E^{n}$ nzymes play a vital role in biology by increasing the rate constants of otherwise slow chemical reactions several orders-of-magnitude. From a fundamental perspective, enzymes function by reducing the energetic barrier separating substrates from products, which corresponds to the breaking and making of chemical bonds (1). In the complex and multifaceted cellular environment $(2,3)$, enzymes must selectively bind their correct substrates among structurally and functionally related molecules that are present at concentrations above or close to their Michaelis constant $\left(K_{\mathrm{M}}\right)$. An example of this fundamental "selectivity problem" is the often strict usage of ATP over GTP as a phosphoryl donor by kinases (4). This preference for ATP has been observed in an experimental analysis of 200 kinases in two human cell lines, where it was found that only a small number of protein kinases also harbor affinity for GTP (5). For the small group of kinases that can utilize GTP as phosphor-donor, it has been found that they can exhibit dual usage of ATP or GTP (6), as in the case of the protein kinase CK2 or strict usage of GTP as in the case of the antibiotic kinase MPH(2')-I (7). The cellular concentrations of both ATP and GTP are of similar orders-ofmagnitude (3 mM and $0.5 \mathrm{mM}$, respectively) (8) and, therefore, enzymes require robust mechanisms to successfully differentiate between these two substrates. In principle, enzymes may use both positive selection for the correct substrate and negative selection, which prevents usage of incorrect substrates, to accomplish this functional property.

Adenylate kinase (Adk) is an essential housekeeping enzyme (9) that controls the energy balance in cells by catalyzing the reversible interconversion of ATP and AMP into two ADP molecules. ATP is turned over in massive amounts and a growing Escherichia coli cell turns over its entire ATP pool several times a second (approximately 1-10 million ATP $\mathrm{s}^{-1}$ ) (10). Because
GTP has specific cellular roles-for example, protein synthesis (11) and signaling in eukaryotes $(12,13)$-it seems likely that Adk has evolved mechanisms to prevent depletion of the GTP pool during phosphorylation of AMP.

Although Adk requires $\mathrm{Mg}^{2+}$ for catalysis, the structural principles underlying Adk function have predominantly been established in the absence of metal ions. Along this line, it has been demonstrated that the binding modes of ADP to Adk from Aquifex aeolicus are virtually identical in the absence or the presence of $\mathrm{Mg}^{2+}$ (14). Adk undergoes dramatic conformational changes in response to binding of its substrates ATP (Fig. $1 A$ and $B)$ and AMP $(15,16)$, which can be probed with various NMR techniques (17-19) and single-molecule FRET experiments $(20,21)$. As a result, Adk has become one of the principal model enzymes to study the relationships between protein structure, conformational dynamics, and catalysis. For example, studies have illuminated that Adk is rate-limited by product release, controlled by slow conformational dynamics (22). The modular Adk structure is composed of a CORE subdomain [required for thermodynamic stability (23)] and flexible ATP and AMP binding subdomains, denoted ATPlid and AMPbd, respectively. The substrate-presenting subdomain, AMPbd, is analogous to peptidebinding motifs of protein kinases, which present a protein substrate

\section{Significance}

As a prerequisite for life, enzymes enhance the rate constants of chemical reactions that otherwise would be orders-of-magnitude too slow. One of the challenges facing enzymes is recognition of correct substrates in the complex and multifaceted cellular environment. This is especially true when distinction must be made between substrates that have similar chemical structures. Here we have discovered an elegant solution to the problem of distinguishing between the correct substrate ATP over the incorrect, but related substrate GTP by the enzyme adenylate kinase. We have found that binding of GTP arrests the enzyme in a catalytically incompetent and open structural state, whereas it is known that binding of ATP activates the enzyme by promoting a large conformational change.

Author contributions: P.R., C.H., U.H.S., and M.W.-W. designed research; P.R., M.R., C.G U.H.S., and M.W.-W. performed research; M.R. contributed new reagents/analytic tools; P.R., C.H., U.H.S., and M.W.-W. analyzed data; and P.R., M.R., C.H., U.H.S., and M.W.-W. wrote the paper.

The authors declare no conflict of interest.

This article is a PNAS Direct Submission.

Published under the PNAS license.

Data deposition: The atomic coordinates and structure factors have been deposited in the Protein Data Bank, www.wwpdb.org (PDB ID code 6F7U).

${ }^{1}$ Present address: Université de Bordeaux/Bordeaux INP, École Nationale Supérieure de Chimie et de Physique de Bordeaux, 33607 Pessac, France; and CNRS, Laboratoire de Chimie des Polymères Organiques (UMR5629), 33607 Pessac, France.

${ }^{2}$ To whom correspondence may be addressed. Email: uwe.sauer@umu.se or magnus wolf-watz@umu.se.

This article contains supporting information online at www.pnas.org/lookup/suppl/doi:10 1073/pnas.1721508115/-/DCSupplemental.

Published online March 5, 2018. 




Fig. 1. ATP dependent activation of Adk. (A) Adk in the substrate-free open state (4AKE) and $(B)$ in the ATP-bound closed state. The closed state shows yeast Adk in complex with the nonhydrolysable ATP analog AMPPCF2P (1DVR) (28). Subdomains are colored according to: ATPlid (blue), AMPbd (green), and CORE (gray). Binding of ATP to Adk activates the enzyme through a conformational change leading to closure of the ATPlid (C) Chemical structure of ATP. The phosphate, ribose and base moieties are colored in orange, gray, and red, respectively. $(D)$ Chemical structure of the guanine base of GTP.

to the active site for subsequent ATP-mediated phosphorylation (4). The large conformational change that occurs for the ATPlid domain is, in fact, an ATP-mediated activation of the enzyme. Here, we present evidence for a mechanism that explains the catalytic ATP versus GTP selectivity of Adk at the atomic level.

\section{Results and Discussion}

Catalytic ATP Vs. GTP Selectivity. A coupled ATPase assay (24) is suitable for quantifying catalytic parameters $\left(k_{\text {cat }}\right.$ and $\left.K_{\mathrm{M}}\right)$ of Adk and other kinases that utilize ATP as their substrate for phosphorylation reactions. In cases where GTP is employed as a phosphoryl donor, the assay is not adequate because the first coupling step would generate ATP by the action of pyruvate kinase. Therefore, ATP will accumulate as GTP is consumed and the resulting Adk activity would be compromised by phosphorylation of AMP from this "secondary" ATP pool. To accurately measure the Adk activity with GTP as the phosphate donor, we used a ${ }^{31} \mathrm{P}$ real-time NMR assay $(25,26)$ that is suitable for quantification of $k_{\text {cat }}$ for phosphoryl donor compounds other than ATP (SI Appendix, Fig. S1). The original assay was modified to function with AMP and triphosphate nucleotides instead of ADP. With this assay, we found that the maximum turnover rate for $E$. coli Adk was reduced to $1.7 \%$ by replacing the phosphoryl donor ATP by GTP. Therefore, Adk exhibits approximately 60fold catalytic selectivity for ATP vs. GTP, a result that supports the notion that Adk has evolved a mechanism to avoid using the intracellular GTP pool in its phosphorylation of AMP. Because the assay does not provide $K_{\mathrm{M}}$ values, we instead obtained $K_{\mathrm{d}}$ values from NMR titration experiments (SI Appendix, Fig. S2). The $K_{\mathrm{d}}$ values for ATP and GTP were found to be $51 \mu \mathrm{M}$ (27) and $109 \mu \mathrm{M}$, respectively, representing an approximate twofold difference in binding affinity. It should be noted that these affinities are considerably lower than the typical millimolar concentrations of cellular ATP and GTP found inside cells (8). Taken together, the significant selectivity that Adk exhibits for ATP over GTP cannot be rationalized by the ability of the enzyme to bind to either one of the two molecules.

Crystal Structure of Adk in Complex with a GTP Analog. The X-ray structure of Adk (Fig. $2 A$ ) in complex with the nonhydrolysable GTP analog $\beta, \gamma$-methyleneguanosine 5'-triphosphate (GMPPCP) (Fig. $2 B$ ) at 1.4- $\AA$ resolution shows an open AdK conformation with a nonproductively bound GTP analog. The position and binding mode of GMP-PCP is radically different from those of ATP (Fig. $3 A$ and $B$ ). A superimposition of the CORE and AMPbd subdomains of the GMP-PCP complex structure and the structure of a complex between yeast Adk and a nonhydrolysable ATP analog (PDB ID code 1DVR) (28) (Fig. 3C) shows that the ATPlid does not close over GMP-PCP. The RMSD between the backbone atoms of the ATPlids in the ATP and GMP-PCP complexes is $8.8 \AA$. Comparing Adk bound to GMP-PCP with substrate free E. coli Adk results in a RMSD of $2.8 \AA$, which establishes that the ATPlid adopts an almost fully open conformation in complex with GMP-PCP (Fig. 3D). Although adopting an open structure in the GMP-PCP complex, the ATPlid has moved minutely toward the closed conformation relative to its orientation observed in the substrate-free state. The structural analysis suggests a mechanism for the low catalytic activity of Adk with GTP-mediated phosphorylation. The large and activating conformational change observed following binding of ATP is not observed in the GMP-PCP complex, wherein Adk is arrested in an open conformation that cannot support phosphorylation of AMP.

Solution Conformation of Adk in Complex with GTP. To establish whether the GMP-PCP bound open Adk structure observed by crystallography is an adequate model for the GTP complex in solution, we used paramagnetic relaxation enhancement (PRE) NMR spectroscopy (29). PRE experiments are suitable for distinguishing between protein conformations that involve largescale structural differences (30). PRE NMR relies on the introduction of a paramagnetic center-for example, an unpaired electron-at a strategic position in a protein. When atoms are in close spatial proximity to the PRE centers, the peak heights of their corresponding NMR resonances are reduced due to increased transverse relaxation rates $\left(\mathrm{R}_{2}\right)$. Inspection of the crystal structures of the open (4AKE) and closed (1AKE) Adk conformations indicated that amino acid Val148 is at a position where attachment of a PRE probe could generate significantly different relaxation rates for residues in the CORE subdomain, depending on whether the ATPlid is open or closed. For quantification of PRE effects in substrate-free, ATP- and GTP-bound Adk complexes, we attached the paramagnetic probe, 2,2,6,6tetramethypiperidin-1-yl oxyl (TEMPO), via a malemide linker, to a cysteine introduced at position 148 (V148C). For substratefree Adk, decreased peak heights resulting from spatial proximity to the TEMPO group were observed predominantly for residues in the ATPlid that are close in sequence to position 148,

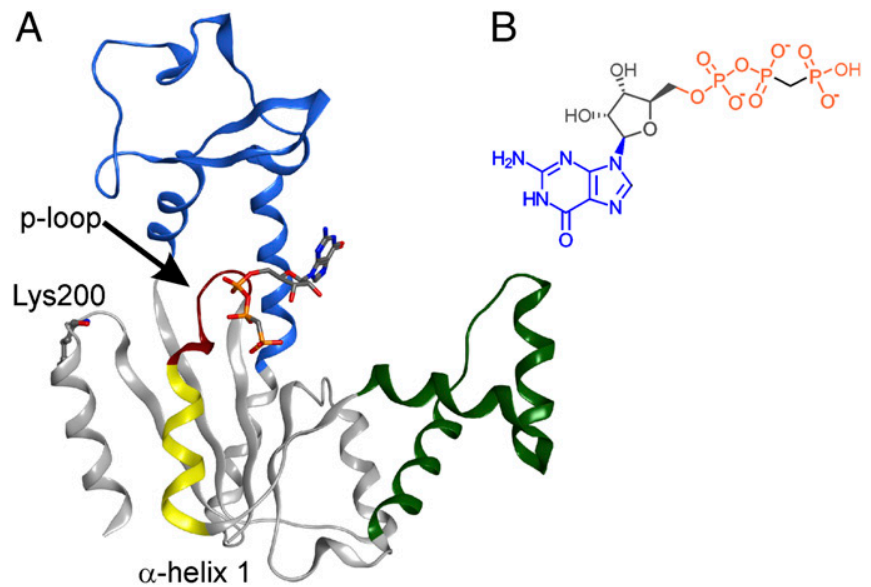

Fig. 2. X-ray structure of $E$. coli Adk in complex with the nonhydrolysable GTP analog GMP-PCP. $(A)$ Ribbon diagram of the structure with 1.4- $\AA$ resolution. Color code: ATPlid (blue), AMPbd (green), CORE (gray), p-loop (dark red), and $\alpha$-helix1 (yellow). The binding of GMP-PCP arrests the ATPlid in an open and catalytically incompetent state. Lys200, important for ATP selectivity, is shown as a ball and stick model. $(B)$ GMP-PCP is a nonhydrolysable GTP analog where the bridging oxygen between the $\beta$ - and $\gamma$-phosphates is replaced with a methylene group. 
A

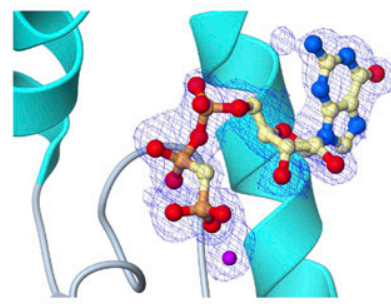

B

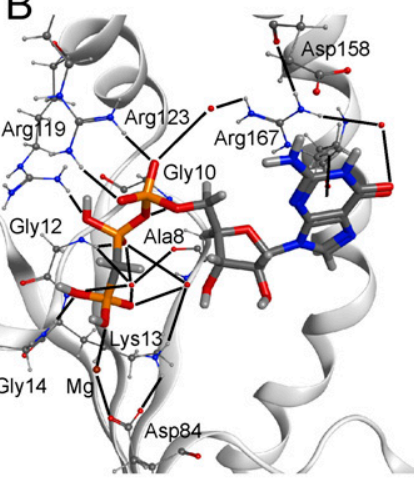

C



D

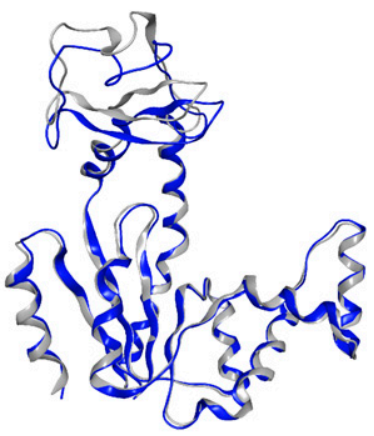

Fig. 3. Arrest of Adk in a catalytically incompetent state in complex with GMP-PCP. (A) Electron density of GMP-PCP clearly defines the position of the compound. $(B)$ Structural details of Adk residues that interact with GMPPCP. Hydrogen bonds are indicated with solid lines. (C) A superimposition of the crystal structures of Adk in complex with GMP-PCP (blue) and yeast Adk bound to the nonhydrolysable ATP analog $\mathrm{AMPPCF}_{2} \mathrm{P}$ (pink) (1DVR) (28) indicates the large conformational change due to ATP binding which activates Adk (for clarity, the nucleotides are not shown). (D) Adk with bound GMP-PCP (blue) is arrested in an open structural state, as is evident from an overlay with substrate free apo-Adk (gray) (4AKE) (15).

and also for several residues of the p-loop (residues 9-15) (Fig. $4 A$ ). It is likely that the reduced peak heights for resonances corresponding to residues of the p-loop are because substratefree Adk dynamically samples closed states $(18,20,21)$. Compared with the substrate-free state of Adk, a dramatic difference was observed for the ATP complex, where reduced peak heights are observed for several residues in $\alpha$-helix 1 of the CORE subdomain (Fig. 4B). The reduced peak-heights for residues in $\alpha$-helix 1 are due to the large-scale conformational change (closing) of the ATPlid following specific binding to ATP. In sharp contrast, there were no changes of peak heights observed for these residues in the GTP complex (Fig. 4C), establishing that Adk adopts an open conformation with GTP. Additional support for our claim that Adk adopts similar open conformations when in complex with either GTP or GMP-PCP comes from the striking similarity of, ${ }^{1} \mathrm{H}-{ }^{15} \mathrm{~N}$ HSQC spectra acquired with saturating concentrations of either nucleotide (SI Appendix, Fig. S3). Cumulatively, both the crystal structure and the NMR analysis in solution show that Adk is arrested in a, catalytically incompetent, open conformational state when bound to GTP. This binding mode provides a robust and protective mechanism against Adk-mediated turnover of the cellular GTP pool.

Adk Interaction with ATP and GTP by Coinciding Residues. Having established that the GMP-PCP complex is a relevant model for rationalizing GTP binding to Adk, the X-ray structure was further analyzed to obtain a detailed understanding of the structural basis for the formation of this catalytically incompetent state. First, the interactions of AdK in complex with ATP are discussed

based on the cocrystal structure determined in the presence of the nonhydrolysable ATP analog AMP-PNP and AMP (1ANK) (31). The conformational change of the ATPlid is triggered by sandwiching ATP between residues from both the ATPlid and the CORE subdomains. The phosphate tail of ATP is recognized through an extensive hydrogen-bonding network involving both residues in the conserved p-loop (Fig. $2 A$ ) segment and three arginines (Arg123, Arg156, and Arg167) of the ATPlid (Fig. 5A). In contrast, the ribose and adenine moieties of ATP are only recognized by a limited set of interactions to Adk. Only two interactions determine the correct orientation of the adenine base: (i) a cation- $\pi$ (or stacking) interaction between Arg119 of the ATPlid and the conjugated $\pi$-system of the adenine base, and (ii) a hydrogen bond donated from the amino group at position $\mathrm{C}^{6}$ of ATP to the backbone carbonyl oxygen of Lys200 in the CORE subdomain. Of these two interactions, only the hydrogen bond donated to Lys 200 has the potential to be selective for ATP over GTP as GTP, with its carbonyl oxygen at position $\mathrm{C}^{6}$, cannot function as a hydrogen bond donor.

Next, the interactions that are responsible for arresting Adk in an open state in complex with GMP-PCP are discussed. The overall interaction pattern is very different from that observed for ATP (Fig. 5B), with the exception of the recognition of the


Fig. 4. Arrest of Adk in an open conformation in complex with GTP. Paramagnetic relaxation enhancements in Adk are displayed for: substratefree as well as ATP- and GTP-bound complexes as quantified from ${ }^{15} \mathrm{~N}$-HSQC spectra. Reduction of normalized peak heights are shown for: $(A)$ substratefree Adk, $(B)$ Adk in complex with ATP, and (C) Adk in complex with GTP. Amino acid residues with a reduction in peak height exceeding two SD from the average decrease are highlighted on the structure of open Adk (4AKE). Reductions in peak heights observed for the ATP but not the GTP complex are highlighted with a dotted oval. This difference reveals that Adk is adopting an open conformation in complex with GTP. 


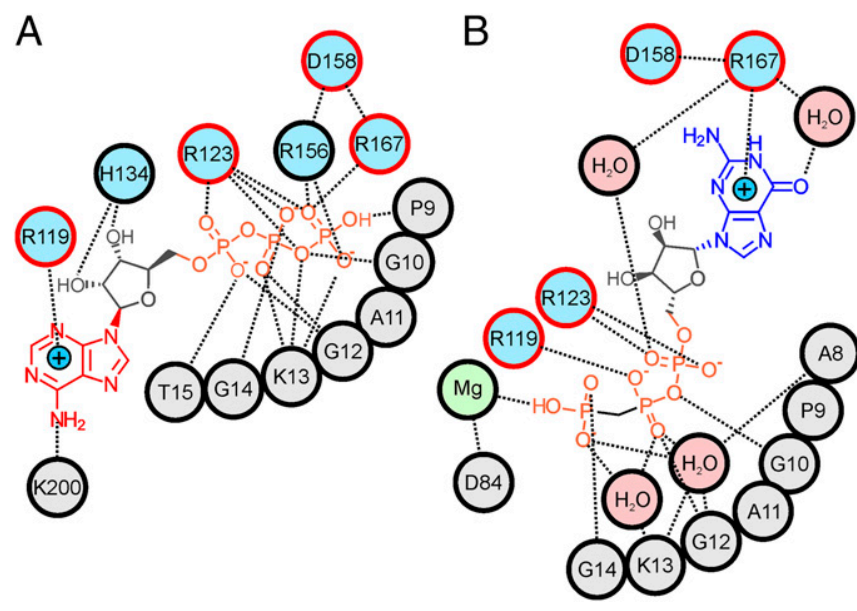

Fig. 5. Schematic illustration of ATP and GMP-PCP binding sites on Adk The binding sites are composed of residues donated from both CORE (gray) and ATPlid (blue) subdomains. $(A)$ The ATP binding site is adapted from the crystal structure of Adk in complex with AMP-PNP (base in red) and AMP (1ANK) (31). (B) Binding of GMP-PCP. In both cases, Arg119, Arg123, Asp158, and Arg167, indicated with blue and red circles, are involved in recognition; however, the modes of ATP (red) GMP-PCP (blue) coordination are different.

phosphate tail by residues in the p-loop. A key interaction is formed between residue Asp158 and the amino group at position $\mathrm{C}^{2}$ of GMP-PCP, which appears to stabilize the open Adk state. Arginine 119 recognizes the phosphate tail of GMP-PCP instead of the base as in the ATP case. Arginine 167 interacts indirectly via water molecules with both the phosphate tail and the carbonyl oxygen at the position $C^{6}$ of the guanosine base. The interaction with the carbonyl oxygen is of particular relevance because it confers binding selectivity toward ATP. In addition, the GMP-PCP complex is stabilized by a cation $-\pi$ interaction between Arg167 and the guanine base. Arg123 interacts with both oxygens of the $\alpha$-phosphate. Finally, we found that Asp84 interacts with a $\gamma$-phosphate oxygen through an indirect interaction with an electron density feature interpreted as a magnesium ion. The coordination of a magnesium by Asp89 in yeast adenylate kinase (equivalent of Asp84 in E. coli adenylate kinase) has previously been demonstrated (32). Taken together, there are four residues from the ATPlid that support the open structure in the GMP-PCP complex: Arg119, Arg123, Asp158, and Arg167. These residues are also important for the correct coordination of ATP. Arg119 recognizes the adenosine base of ATP with a cation $-\pi$ interaction, as discussed above. Arg123 interacts with the phosphate tail also in ATP but while only the $\alpha$-phosphate is recognized in the GMP-PCP complex, both the $\alpha$ - and $\gamma$-phosphate oxygens are recognized in the ATP complex. Asp158 interacts indirectly with ATP via hydrogen bonding to arginines 156 and 167 that are in direct contact with the phosphate tail. Taken together the interaction patterns for Adk binding to ATP and GMP-PCP demonstrates that the two distinctly different binding modes are partially mediated by a set of overlapping residues. Therefore, it appears that the positive selection of ATP and the negative selection of GTP has emerged by evolution of dual functionality of a set of key Adk residues.

Structure-Function Relationship of a Key Hydrogen Bond in ATP Recognition. The structural analysis described above suggests that the hydrogen bond donated from the amino group at position $\mathrm{C}^{6}$ of ATP to the backbone oxygen of Lys 200 of Adk is essential for correct binding orientation of the substrate. This mode of ATP recognition is a conserved feature of the Adk enzyme family found throughout evolution from bacteria to human (SI Appendix, Table S1). Furthermore, in a systematic analysis of the eight groups of eukaryotic protein kinases (33), we found that the recognition mode is utilized also in this important family of enzymes (SI Appendix, Table S2). Examples of a hydrogen bond interaction between a backbone carbonyl oxygen and the amino group at position $\mathrm{C}^{6}$ of ATP is shown in SI Appendix, Fig. S4 for the human protein kinases cAMP-dependent protein kinase A (4WB5) (34), insulin receptor kinase (1IR3) (35), and Abl kinase (2G1T) (36).

Taking these data together, it is evident that the recognition of the adenine base of ATP through the mentioned hydrogen bond is an important feature of kinase function. To test the hypothesis that the hydrogen bond alone is sufficient for selectivity of Adk toward nucleotide triphosphates, a structure-function analysis was performed, utilizing both commercially available and novel synthetic nucleotide triphosphates that differ in their hydrogen bond donating capacity at position $\mathrm{C}^{6}$. The analysis of these analogs was benchmarked against the activities and binding affinities determined for ATP and GTP. As described above, these two nucleotides bind to Adk with the ATPlid in closed and open conformations, respectively (Fig. 3C). Inosine triphosphate (ITP) differs from ATP because it contains a carbonyl functionality at $\mathrm{C}^{6}$ instead of an amine (Fig. 6). This analog exhibited a relative activity of $1.0 \pm 0.2 \%$ and a $K_{\mathrm{d}}$ value of around $300 \mu \mathrm{M}$. The activity is very similar to that of GTP and indicates that ITP also binds to Adk in an open conformation. The increase in $K_{\mathrm{d}}$ observed for ITP relative to GTP is fully consistent with an open binding mode that is destabilized relative to the GTP complex due to the inability of ITP to donate a hydrogen bond to Asp158 (ITP lacks the $\mathrm{C}^{2}$ amine substituent of GTP). A structural analysis of the Adk:ITP complex was performed by comparing ${ }^{1} \mathrm{H}-{ }^{15} \mathrm{~N}$ HSQC spectra of Adk with saturating amounts of ITP and GTP (SI Appendix, Fig. S5). Because the spectra are virtually identical, it appears that Adk adopts an open conformation also in complex with ITP. Compound 1 (Fig. 6) lacks the $\mathrm{C}^{6}$ amine functionality of ATP (replaced by a hydrogen), thereby completely removing the hydrogen bond donating capacity at this position. The activity of compound 1 is in-between the values observed for ATP and GTP and is roughly sixfold lower in comparison with ATP (Table 1). Conversely, the $K_{\mathrm{d}}$ for binding of compound 1 to Adk is approximately sixfold higher relative to the value for ATP. These characteristics indicate that Adk can indeed close the ATPlid over compound 1 to support catalysis but that the fraction of ATPlid in the closed state is around sixfold lower in comparison with the scenario with ATP. This result illustrates that the selectivity for ATP over GTP does not only depend on the removal of hydrogen bonding capacity at $\mathrm{C}^{6}$, but also on a negative contribution caused by interference of the $\mathrm{C}^{6}$ carbonyl of GTP with the backbone carbonyl of Lys200. Therefore, formation of the catalytically incompetent GTP complex depends on specific interactions (residues Arg119, Arg123, Asp158, and Arg167) but also on a negative contribution from the mentioned interference. The activity and


Fig. 6. Compounds for structure-activity relationships. The compounds were selected to probe the importance of the hydrogen bond formed between the $\mathrm{NH}_{2}$ group at position $\mathrm{C}^{6}$ in ATP and the backbone carbonyl oxygen of Lys 200 in Adk. While ATP has a hydrogen bond donor at position $\mathrm{C}^{6}$, ITP has a hydrogen bond acceptor (carbonyl oxygen) in that position. Compound 1 lacks a substituent at position $C^{6}$, while compound 2 is a "rescue variant" of compound 1, where the hydrogen bonding capacity is reintroduced with a hydroxyl group. 
Table 1. Activities and binding affinities

\begin{tabular}{lcc} 
Substrate & Activity relative to ATP, $\%$ & $K_{\mathrm{d}}$ \\
\hline ATP & 100 & $51 \pm 13 \mu \mathrm{M}$ (ref. 27) \\
GTP & $1.7 \pm 0.5$ & $109 \pm 16 \mu \mathrm{M}$ \\
ITP & $1.0 \pm 0.2$ & $292 \pm 100 \mu \mathrm{M}$ \\
Compound 1 & $16.3 \pm 2.8$ & $330 \pm 8 \mu \mathrm{M}$ \\
Compound 2 & $98.0 \pm 16.2$ & $35 \pm 9 \mu \mathrm{M}$
\end{tabular}

binding affinity displayed by compound 1 could be rescued to ATP levels by introducing hydrogen bond donor capacity at position $\mathrm{C}^{6}$ with a hydroxyl group, while replacing the pyrimidine motif of the adenine ring with a simple phenyl component (compound 2) (Fig. 6). Compound 2 displayed activity and $K_{\mathrm{d}}$ values that were indistinguishable from those of ATP, establishing that the hydrogen bond between the amine group at position $\mathrm{C}^{6}$ of ATP and the backbone carbonyl of Lys200 is crucial for recognition and turnover of ATP by Adk. Taking these data together, we find that the structure-function analysis described here, as well as the conservation of the key hydrogen bond across the family of human protein kinases, suggest that formation of a hydrogen bond between a backbone carbonyl and the substituent at position $\mathrm{C}^{6}$ of ATP is of fundamental importance for selectivity.

\section{Conclusions}

The ability of enzymes to utilize the correct substrate in a highly selective manner is a fundamental and critical characteristic that allows them to function in complex cellular environments. Our work presents evidence for a mechanism that rationalizes the selectivity of Adk for ATP over GTP. While productive recognition of ATP induces a large and activating conformational change of the ATPlid, binding to GTP arrests Adk in an open and catalytically incompetent state (Fig. 7). ATP and GTP bind to Adk in distinctly different orientations with contacts that, in part, are mediated by a set of coinciding amino acids. Accordingly, these residues can be considered as "gatekeepers," which influence both the positive selection of ATP and, at the same time, negative selection of GTP. Our structure-function analysis of ATP analogs revealed that specific selection of ATP depends on a key hydrogen bond formed between the amino group at position $\mathrm{C}^{6}$ of ATP and the backbone carbonyl oxygen of Lys 200 . It is likely that the importance of backbone-mediated ATP
A
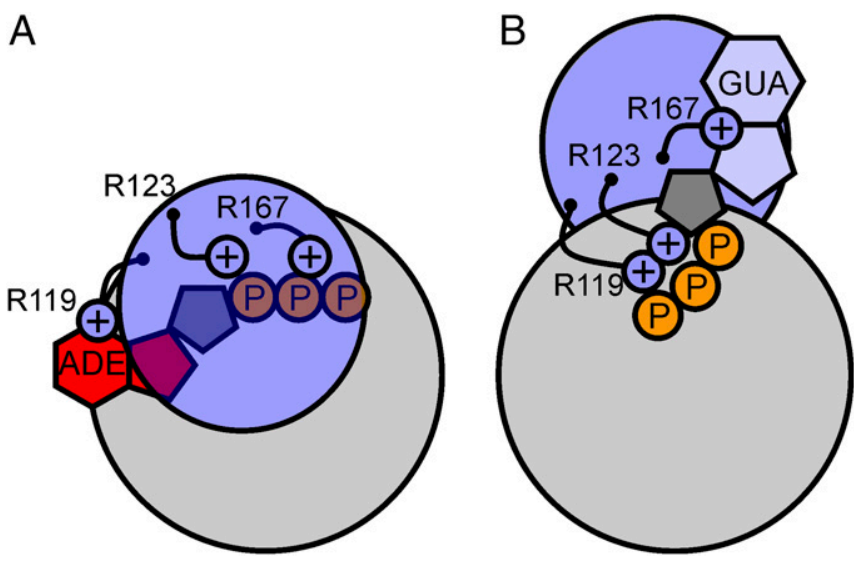

Fig. 7. Schematic model for catalytic selectivity of ATP versus GTP for Adk. Binding of ATP $(A)$ leaves Adk in a catalytically competent state with a closed ATPlid, while GTP $(B)$ binding arrests the enzyme in an open and catalytically incompetent state. Residues with dual binding functionalities that underlie positive selection of ATP and negative selection of GTP are indicated. For clarity, the AMP binding domain is not shown. ADE, adenosine; GUA, guanosine.
Table 2. Crystallographic data collection and refinement statistics for $6 \mathrm{~F} 7 \mathrm{U}$

Parameters

Adk:GMP-PCP complex

Data collection

Wavelength, ^

Space group (Nr.)

eam line ID23-1 ESRF, France

0.97939

Unit cell dimensions

$$
a, b, c, \AA
$$

$$
\alpha, \beta, \gamma,{ }^{\circ}
$$

Molecules per asymmetric unit

Resolution range (highest shell), $\AA$

Accepted reflections

Unique reflections

Completeness, \%

Multiplicity

Mean (I/бI)

$R_{\text {merge, } \%}$

$R_{\text {meas, }} \%$

$R_{\text {pim }}, \%$

$\mathrm{CC}_{1 / 2}, \%$

CC* $\%$

Wilson B-factor, $\AA^{2}$

$132.88,31.78,54.2$

$90.0,112.35,90.0$

1

49.04-1.07 (1.13-1.07)

$390,556(53,864)$

$96.3(92.2)$

$4.4(4.2)$

$11.2(1.42)$

$5.2(99.0)$

$5.9(113.2)$

$3.6(63.3)$

$99.8(70.7)$

$99.9(94.7)$

12.9

Refinement

Resolution range (highest shell), $\AA$

Reflections used for refinement

Reflections used for $R_{\text {work }}$

Reflections used for $R_{\text {free }}$

$R_{\text {work, }} \%$

$R_{\text {free, }} \%$

Overall map CC (Fc, 2mFo - DFc), \%

RMSD

Bond length, $\AA$

Bond angles,

Ramanchandran plot statistics

Residues in favored region, \%

Residues in allowed region, $\%$

Residues in outlier region, \%

All-atom clash score

Total number of atoms

No. of nonhydrogen atoms

Protein (nonhydrogen)

GMP-PCP

$\mathrm{Mg}^{2+}$

Solvent $\left(\mathrm{H}_{2} \mathrm{O}\right)$ (nonhydrogen)

Average B-factors, $\AA^{2}$

All atoms

Protein chains

$\mathrm{Mg}^{2+}$

33.11-1.40 (1.47-1.40)

$41,048(3,204)$

$40,138(5,638)$

915 (129)

17.8 (20.1)

$19.0(22.4)$

87.5

0.005

0.803

98.7

1.4

0.4

3.3

3,900

2,094

1,754

49

1

306

29.0

25.1

27.6

37.5

Water molecules
$89,469(12,823)$

Values in parentheses are for the highest resolution shell. $\mathrm{Nr}$, number index.

selectivity is of a general nature, as it represents a conserved structural motif in the family of human protein kinases. However, absence of the hydrogen bond is not sufficient for arrest of GTP in the catalytically incompetent state. The catalytically incompetent Adk-GMP-PCP complex is further stabilized by interactions between residues Asp158 and Arg167 and groups on the guanine base and is also promoted by negative interference between the carbonyl oxygen and the backbone carbonyl oxygen of Lys200. As discussed several of the interactions between Adk and GMP-PCP are mediated by water molecules. It has previously been shown that the capacity of protein kinase CK2 to use both ATP and GTP as phosphoryl donors is dependent on water molecules that enable binding of GTP to the active 
site (6). It therefore seems that, from an evolutionary perspective, binding of different molecules to an active site can be enabled by incorporation of water molecules.

We propose a potential biological role that rationalizes why Adk has evolved such a sophisticated mechanism to discriminate between ATP and GTP. GTP is required for a number of specific cellular functions and the selectivity of Adk minimizes the depletion of the cellular GTP pool, while maintaining energy homeostasis. An intriguing aspect of our findings is related to the relevance of the large conformational change observed for Adk in response to ATP binding. Conformational changes in enzymes have several functions, including: removal of water molecules from active sites to prevent nonproductive side reactions (37), to allow proper alignment of substrate molecules to facilitate chemical transformations, and for the purpose of activating functional groups on substrate molecules (1). Here, we have discovered that the large-scale conformational change in Adk is utilized also to accommodate catalytic selectivity toward GTP simply by formation of a stable complex between the open Adk conformation and GTP. Hence, the selectivity mechanism discovered here adds the unexpected finding that activating conformational changes also can be utilized by an enzyme to avoid using an incorrect substrate during catalysis. Because the conformational change is a dynamic event, our findings points toward a coupling between enzyme dynamics and substrate selectivity. Hence, our work underlines the crucial role of dynamics for the proper function of enzymes.

1. Fersht AR (1999) Structure and Mechanism in Protein Science (W. H. Freeman, New York).

2. McGuffee SR, Elcock AH (2010) Diffusion, crowding \& protein stability in a dynamic molecular model of the bacterial cytoplasm. PLoS Comput Biol 6:e1000694.

3. Mu X, et al. (2017) Physicochemical code for quinary protein interactions in Escherichia coli. Proc Natl Acad Sci USA 114:E4556-E4563.

4. Huse M, Kuriyan J (2002) The conformational plasticity of protein kinases. Cell 109 275-282.

5. Becher I, et al. (2013) Affinity profiling of the cellular kinome for the nucleotide cofactors ATP, ADP, and GTP. ACS Chem Biol 8:599-607.

6. Niefind K, Pütter M, Guerra B, Issinger OG, Schomburg D (1999) GTP plus water mimic ATP in the active site of protein kinase CK2. Nat Struct Biol 6:1100-1103.

7. Shakya T, Wright GD (2010) Nucleotide selectivity of antibiotic kinases. Antimicrob Agents Chemother 54:1909-1913.

8. Traut TW (1994) Physiological concentrations of purines and pyrimidines. Mol Cell Biochem 140:1-22.

9. Tükenmez H, Magnussen HM, Kovermann M, Byström A, Wolf-Watz M (2016) Linkage between fitness of yeast cells and adenylate kinase catalysis. PLoS One 11:e0163115.

10. Lane N, Martin W (2010) The energetics of genome complexity. Nature 467:929-934

11. Liljas A, Ehrenberg M (2013) Structural Aspects of Protein Synthesis (World Scientific Publishing, London), 2nd Ed.

12. Bourne HR, Sanders DA, McCormick F (1990) The GTPase superfamily: A conserved switch for diverse cell functions. Nature 348:125-132.

13. Cherfils J, Zeghouf M (2011) Chronicles of the GTPase switch. Nat Chem Biol 7: 493-495.

14. Kerns SJ, et al. (2015) The energy landscape of adenylate kinase during catalysis. Nat Struct Mol Biol 22:124-131.

15. Müller CW, Schlauderer GJ, Reinstein J, Schulz GE (1996) Adenylate kinase motions during catalysis: An energetic counterweight balancing substrate binding. Structure 4:147-156.

16. Müller CW, Schulz GE (1992) Structure of the complex between adenylate kinase from Escherichia coli and the inhibitor Ap5A refined at $1.9 \mathrm{~A}$ resolution. A model for a catalytic transition state. J Mol Biol 224:159-177.

17. Kovermann $\mathrm{M}$, et al. (2015) Structural basis for catalytically restrictive dynamics of a high-energy enzyme state. Nat Commun 6:7644.

18. Kovermann M, Grundström C, Sauer-Eriksson AE, Sauer UH, Wolf-Watz M (2017) Structural basis for ligand binding to an enzyme by a conformational selection pathway. Proc Natl Acad Sci USA 114:6298-6303.

19. Adén J, Wolf-Watz M (2007) NMR identification of transient complexes critical to adenylate kinase catalysis. J Am Chem Soc 129:14003-14012.

20. Henzler-Wildman KA, et al. (2007) Intrinsic motions along an enzymatic reaction trajectory. Nature 450:838-844.

21. Hanson JA, et al. (2007) Illuminating the mechanistic roles of enzyme conformational dynamics. Proc Natl Acad Sci USA 104:18055-18060.

\section{Materials and Methods}

GTP, ITP, ATP, AMP, and GMP-PCP were purchased from Sigma-Aldrich. The nucleotides were used without further purification. Compounds 1 and 2 were synthesized as a part of this study.

E. coli adenylate kinase was overexpressed and purified following a previously published method (38). For paramagnetic relaxation enhancement measurements a malemide-TEMPO (Merck), free-radical probe was attached to an introduced cysteine at position 148 (V148C) in the ATPlid. The Adk variant was expressed as described above and further purified under reducing conditions ( $1 \mathrm{mM}$ TCEP). The PRE probe was attached to Adk by adding a fivefold molar excess of malemide-TEMPO to an enzyme solution in an oxidizing environment. After $10 \mathrm{~min}$, the reaction was quenched by the addition of $\beta$-mercaptothanol to a final concentration of $10 \mathrm{mM}$. The protein with the covalently attached probe was then purified by size exclusion chromatography using a HiPrep 26/60 Sephacryl S-100 HR column (Amersham). Mass spectroscopy was used to quantify the conjugation process.

Description of materials and methods for NMR spectroscopy, X-ray crystallography and synthetic organic chemistry can be found in the SI Appendix. See Table 2 for crystallographic data collection and refinement statistics for 6F7U.

ACKNOWLEDGMENTS. We thank The Protein Expertise Platform at Umeå University for providing reagents and expertise for protein overexpression and purification. NMR experiments were performed at "NMR for life" at Umeå University. This study was financially supported by grants from The Swedish Research Council (to M.W.-W. and C.H.), The Kempe Foundation (to M.W.-W.), and Knut and Alice Wallenberg Foundation Grant KAW 2013.0187 (to C.H.).

22. Wolf-Watz M, et al. (2004) Linkage between dynamics and catalysis in a thermophilicmesophilic enzyme pair. Nat Struct Mol Biol 11:945-949.

23. Rundqvist $L$, et al. (2009) Noncooperative folding of subdomains in adenylate kinase. Biochemistry 48:1911-1927.

24. Rhoads DG, Lowenstein JM (1968) Initial velocity and equilibrium kinetics of myokinase. J Biol Chem 243:3963-3972.

25. Rogne P, Sparrman T, Anugwom I, Mikkola J-P, Wolf-Watz M (2015) Realtime 31P NMR investigation on the catalytic behavior of the enzyme adenylate kinase in the matrix of a switchable ionic liquid. ChemSusChem 8:3764-3768.

26. Rogne $P$, Wolf-Watz M (2016) Urea-dependent adenylate kinase activation following redistribution of structural states. Biophys J 111:1385-1395

27. Olsson U, Wolf-Watz M (2010) Overlap between folding and functional energy landscapes for adenylate kinase conformational change. Nat Commun 1:111.

28. Schlauderer GJ, Proba K, Schulz GE (1996) Structure of a mutant adenylate kinase ligated with an ATP-analogue showing domain closure over ATP. J Mol Biol 256: 223-227.

29. Clore GM, Iwahara J (2009) Theory, practice, and applications of paramagnetic relaxation enhancement for the characterization of transient low-population states of biological macromolecules and their complexes. Chem Rev 109:4108-4139.

30. Battiste JL, Wagner G (2000) Utilization of site-directed spin labeling and highresolution heteronuclear nuclear magnetic resonance for global fold determination of large proteins with limited nuclear overhauser effect data. Biochemistry 39: 5355-5365.

31. Berry MB, et al. (1994) The closed conformation of a highly flexible protein: The structure of $E$. coli adenylate kinase with bound AMP and AMPPNP. Proteins 19: 183-198.

32. Abele U, Schulz GE (1995) High-resolution structures of adenylate kinase from yeast ligated with inhibitor Ap5A, showing the pathway of phosphoryl transfer. Protein Sci 4:1262-1271

33. Manning G, Whyte DB, Martinez R, Hunter T, Sudarsanam S (2002) The protein kinase complement of the human genome. Science 298:1912-1934.

34. Cheung J, et al. (2015) Structural insights into mis-regulation of protein kinase $A$ in human tumors. Proc Natl Acad Sci USA 112:1374-1379.

35. Hubbard SR (1997) Crystal structure of the activated insulin receptor tyrosine kinase in complex with peptide substrate and ATP analog. EMBO J 16:5572-5581.

36. Levinson NM, et al. (2006) A Src-like inactive conformation in the Abl tyrosine kinase domain. PLoS Biol 4:e144

37. Ådén J, Weise CF, Brännström K, Olofsson A, Wolf-Watz M (2013) Structural topology and activation of an initial adenylate kinase-substrate complex. Biochemistry 52: 1055-1061.

38. Reinstein J, Brune M, Wittinghofer A (1988) Mutations in the nucleotide binding loop of adenylate kinase of Escherichia coli. Biochemistry 27:4712-4720. 